\title{
4.3 Gesundheitsförderung
}

Petra Kolip, Thomas Abel

Gesundheitsförderung schafft Lebensbedingungen, in denen sich Menschen gesund entwickeln können. Ein wichtiger Punkt hierbei ist die Stärkung der persönlichen Kompetenzen.

In diesem Abschnitt erörtern wir zuerst die wichtigsten Perspektiven, Definitionen und Konzepten im Bereich der Gesundheitsförderung. Anschließend beschäftigen wir uns ausführlicher mit den beiden gesundheitsfördernden Settings Krankenhaus und Schule.

Schweizerische Lernziele: CHP 28, CPH 35, CHP 38

Anders als die Krankheitsprävention konzentriert sich die Gesundheitsförderung in erster Linie auf die Schaffung von gesundheitsförderlichen Lebensbedingungen und Ressourcen. Im Bereich Public Health ergänzen sich die beiden Ansätze und verfolgen dabei die gleiche Zielsetzung, nämlich Gesundheit und Lebensqualität von möglichst vielen Menschen zu erhöhen. Was aber genau ist Gesundheitsförderung? Gesundheitsförderung hat zum Ziel, die sozialen und individuellen Lebensbedingungen so zu gestalten, dass Menschen darin möglichst viele internale (im Individuum verankerte) und externale (außerhalb des Individuums gelegene) Ressourcen für eine gesunde Lebensgestaltung zur Verfügung haben. Die Ziele der Gesundheitsförderung sind geprägt von spezifischen Werten. Grundlegende Werthaltung hierbei ist die der Fairness (Equity; vgl. Kap. 1.6). Als Zielvorstellung gilt der pro-aktiv handelnde Mensch, der sich für seine Gesundheit und die Gesundheit der Gemeinschaft einsetzen kann. Diese Vorstellungen finden sich auch in der Ottawa $\mathrm{Charta}^{16}$, die die Ziele der Gesundheitsförderung folgendermaßen definiert:

"Gesundheitsförderung zielt auf einen Prozeß, allen Menschen ein höheres Maß an Selbstbestimmung über ihre Gesundheit zu ermöglichen und sie damit zur Stärkung ihrer Gesundheit zu befähigen."

Gesundheitsförderung richtet den Blick primär auf soziale Faktoren und Prozesse, die sich auf Gesundheit, Lebensqualität und Wohlbefinden auswirken können. Diese Faktoren und Prozesse sind direkt durch die Lebensverhältnisse und das Handeln der Menschen in ihren jeweiligen Lebensverhältnissen bestimmt. Sie können sozialer, kultureller, politischer und ökonomischer Natur sein und bestimmen die materiellen und nicht-materiellen Lebensbedingungen und Ressourcen für die Gesundheit der Menschen sowie für ihr Gesundheitsverhalten und -erleben. Gesundheitsrelevante Faktoren und Prozesse sind auf allen gesellschaftlichen Ebenen wirksam (s. Abb. 4.1). Sie selbst werden von gesellschaftlichen Kräften wie der gesundheitsrelevanten Politik (z.B. in den Bereichen Gesundheit, Umwelt und Verkehr), von den vorhandenen Schulsystemen, Arbeits- und Wohnungsmarktbedingungen ebenso beeinflusst wie von den Le-

16 Die Ottawa-Charta zur Gesundheitsförderung wurde im Jahr 1986 von der WHO anlässlich der Ersten Internationalen Konferenz zur Gesundheitsförderung im kanadischen Ottawa diskutiert und verabschiedet. 
bensbedingungen in einem Quartier oder von der Zugehörigkeit zu einer Religionsgemeinschaft. Menschen handeln in diesen Systemen und Strukturen, sie stabilisieren oder verändern sie aber auch. Die Lebensbedingungen auf diesen Ebenen (s.a. Soziale Determinanten, Kap. 4.1) prägen die Chancen der Menschen auf eine gute Gesundheit. Dies bedeutet, dass die sozialen Kontexte den Menschen Ressourcen zur Verfügung stellen, die mehr oder weniger zu ihrer Gesunderhaltung beitragen und zu mehr oder weniger Risikoexpositionen führen.

Gesundheitsförderung will nun die Lebensräume der Menschen so verbessern, dass sie ihre Gesundheitspotenziale möglichst optimal ausschöpfen können. Lebensräume, die sich im Hinblick auf ihre gesundheitsrelevanten Bedingungen und Interventionspotentiale abgrenzen bzw. nutzen lassen, werden als Settings bezeichnet. Solche Settings sind beispielsweise Wohnquartiere, Schulen und Betriebe. Die betriebliche Gesundheitsförderung fragt z. B. nach förderlichen Einflüssen auf die Gesundheit der Menschen im Setting Betrieb (s. Kap. 6.5), indem sie Führungskräfte darin schult, soziale Unterstützung zu fördern und mit verschiedenen Arbeitszeitmodellen die Handlungsspielräume für Arbeitnehmer zu erhöhen. Gesundheitsförderung im Betrieb geht also weit über die engere, am medizinischen Krankheitsbegriff orientierte Prävention und auch über den Arbeitsschutz hinaus. Ihr Ziel ist es, sowohl vor Ort spezifische Verbesserungen zu erreichen als auch breite strukturelle Änderungen zu verwirklichen. Beispiele hierfür wären etwa die dauerhafte Verankerung von Arbeitnehmermitbestimmungsrechten im Hinblick auf gesundheitsrelevante Bedingungen im Betrieb, die Einführung von fettund salzreduzierten Menüs in der Kantine, das Angebot einer Rückenschule oder die Verhinderung von Unfällen im Betrieb (s.a. Kap. 6.1).

Gesundheitsförderung betrachtet den Menschen aus salutogenetischer Perspektive (s. Kap. 1.3.1). Im Zentrum von Forschung und Interventionen stehen dabei die Prozesse der Entstehung und Erhaltung von Gesundheit (s. a. Stress, Kap. 4.4.2). Gesundheit wird hier als ein dynamischer Prozess verstanden, bei dem sich der Mensch ständig zwischen den beiden Polen eines Kontinuums bewegt. Auf der einen Seite dieses Kontinuums liegen vollständiges Wohlbefinden und umfassende Leistungs- und Entwicklungsfähigkeit, auf der anderen Seite weitestgehende Einschränkungen und letztendlich der Tod (Abb. 4.3).

Die Bezeichnung Salutogenese (s. Kap. 1.3.1 und Kap. 4.1) bildet dabei den perspektivischen Kontrast zur Pathogenese: Es geht hier nicht primär darum zu klären, was Menschen krank macht, sondern in erster Linie um die Frage: Was hält Menschen trotz Risiken und Belastungen - gesund? Im Prozess der Salutogenese hin zu mehr

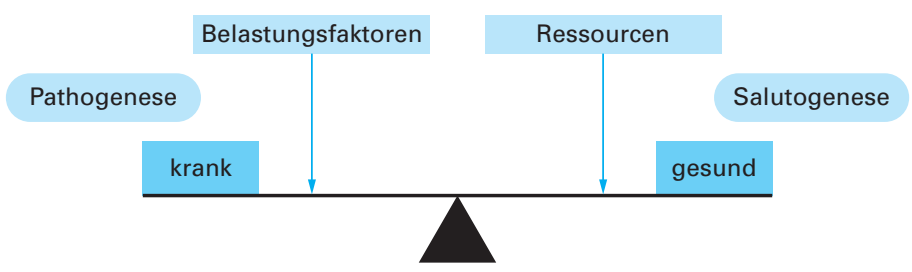

Abb. 4.3: Wechselbeziehung zwischen Salutogenese und Pathogenese. Die Abbildung stellt das Kontinuum zwischen den Polen Gesundheit und Krankheit als kontinuierlichen, dynamischen Prozess dar, der ständig durch eine Vielzahl von Belastungsfaktoren und Ressourcen beeinflusst wird. 
Gesundheit sind die materiellen und nicht-materiellen gesundheitsrelevanten Ressourcen (wie z.B. gesunde Wohnbedingungen, hinreichendes Einkommen, soziale Netzwerke, Gesundheitswissen), über die Menschen verfügen, von entscheidender Bedeutung. Wie zahlreiche Studien zeigen konnten, sind diese Ressourcen in der Bevölkerung und in Subpopulationen oftmals sehr ungleich verteilt. Die unteren sozialen Schichten sind hierbei meist benachteiligt. Gesundheitsförderung hat dabei auch immer einen politischen und emanzipatorischen Anspruch. Interventionen der Gesundheitsförderung sind stets auf mehr Chancengleichheit ausgerichtet. Die Gesundheitsförderung setzt dabei häufig Methoden des Empowerments („Ermächtigung“) und der Partizipation auf allen Ebenen und mit allen Beteiligten ein (s.a. Kap. 1.3.2). Damit ist sie bestrebt, ihren Leitwerten gerecht zu werden und zugleich nachhaltigere Erfolge zu erzielen. So zeigen Forschungsergebnisse, dass Verbesserungen in den Lebensverhältnissen und Verhaltensweisen der Menschen längerfristig mehr Wirkung erzielen, wenn sie unter Mitwirkung der Betroffenen geschaffen wurden (s. Box 4.3.1).

Box 4.3.1: Frauengesundheit im Bremer Stadtteil Tenever.

Ein Beispiel für eine Gesundheitsförderung, die die schichts- und genderspezifischen Bedürfnisse der Bevölkerung in den Vordergrund stellt, sind die gemeindebezogenen Aktivitäten im Bremer Stadtteil Tenever. In diesem sozial benachteiligten Teil der Hansestadt wurde bereits vor 22 Jahren der Frauengesundheitstreff Tenever (heute: Frauengesundheit in Tenever) eingerichtet, der sich der niederschwelligen Arbeit verpflichtet hat. Da die Besucherinnen dort häufig über

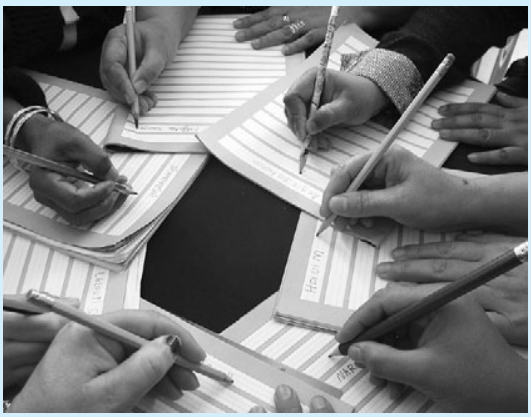
geringe Deutschkenntnisse verfügen und zudem kaum Lesen und Schreiben können, wurden Alphabetisierungs- und Deutschkurse angeboten. Nach einiger Zeit formulierten die Frauen in diesen Kursen auch den Wunsch, Fahrradfahren zu erlernen, da das Bremer Stadtzentrum $8 \mathrm{~km}$ entfernt liegt und Fahrradfahren ein Teil der Bremer Stadtkultur ist. Eine wissenschaftliche Evaluation konnte schließlich zeigen, dass es aufgrund der Angebote zu einer nachhaltigen Verbesserung der Partizipation, des Selbstwertgefühls und der gesundheitsförderlichen Mobilität bei der sozial benachteiligten Bevölkerung gekommen war. Die Frauen sind mittlerweile im Stadtteil sehr gut vernetzt. Über persönliche Beziehungen wird ihr Kreis immer größer.

Copyright der Abbildung: Frauengesundheit in Tenever

Es geht in der Gesundheitsförderung also letztlich um zwei voneinander abhängige und ineinander wirkende Prozesse:

- die Veränderung der gesellschaftlichen Bedingungen mit dem Ziel der Verbesserung gesundheitsrelevanter Lebensbedingungen und

- die Befähigung der Menschen, sich für gesunde Lebensbedingungen einzusetzen und eigene gesündere Verhaltensmuster umzusetzen. 
Die Gesundheitsförderung setzt dabei schwerpunktmäßig auf die Stärkung spezifischer sozialer und individueller Ressourcen (s. Kap. 4.1).

\section{Settings: Krankenhaus und Schule}

Angeregt durch die Ottawa Charta (1986) wurde weltweit in die Entwicklung von gesundheitsförderlichen Settings investiert. Gleichzeitig wurde die Bildung von kooperativen Netzwerken in diesem Bereich unterstützt. Beispiele für solche Settings sind Städte und Quartiere, Schulen und Betriebe, aber auch Krankenhäuser.

Krankenhäuser: Krankenhäuser, die sich dem Netzwerk Health Promoting Hospitals angeschlossen haben, nehmen alle Gruppen, die sich in diesem Setting bewegen, gleichermaßen ins Blickfeld: PatientInnen (inkl. der Angehörigen), Pflegepersonal, ÄrztInnen ebenso wie das Verwaltungspersonal. Ihr Ziel ist es, die Organisation „Krankenhaus" so weiterzuentwickeln, dass durch eine Veränderung der Arbeitsbedingungen (insbesondere der Routinetätigkeiten) für alle Beteiligten mehr Gesundheit möglich wird. Um dabei dem Anspruch der Partizipation gerecht zu werden, muss dieser Gestaltungsprozess immer unter Einbeziehung der PatientInnen, aber auch des Personals zustande kommen. Die Problemanalyse und die Erarbeitung der Entwicklungsschritte erfolgt dabei typischerweise im Rahmen von so genannten Gesundheitszirkeln. Damit sind Austausch- und Kooperationsforen gemeint, die sich aus den jeweils betroffenen Beschäftigten zusammensetzen und für deren Arbeitsbereiche verbindliche Veränderungen vereinbart werden. Aufgabe der Teilnehmerlnnen an diesen Zirkeln ist es, jeweils für ihren Arbeitsbereich aus der Sicht der Betroffenen die vorhandenen gesundheitlichen Probleme zu formulieren und Verbesserungsvorschläge zu erarbeitet. Die konkreten Interventionen der Gesundheitsförderung im Krankenhaus können sehr unterschiedlich sein. Sie reichen von einer veränderten Krankenhausverpflegung über die Schaffung von Ruheräumen bis hin zur Aktivierung der PatientInnen. Auch die Stärkung der PatientInnen in Fragen des gesundheitsförderlichen Handelns und der Patientensicherheit (s. Kap. 3.4) gehören hierzu, ebenso wie Veränderungen in der Arbeitsorganisation sowie eine verbesserte Kommunikation unter allen Beteiligten.

Schulen: Ein ähnlicher Prozess lässt sich für das Setting Schule beschreiben. Gesundheitsfördernde Schulen forcieren einen Schulentwicklungsprozess, der den Lern- und Arbeitsplatz Schule gesundheitsfördernd gestaltet. Auch hier geht es darum, möglichst alle Beteiligten, d. h. Lehrkräfte, Schülerlnnen sowie nicht-lehrendes Personal in diesen Prozess einzubinden, um eine gesundheitsförderliche Entwicklung für sie und mit ihnen in Gang zu setzen. Eine settingbezogene Gesundheitsförderung beinhaltet dabei z. B. nicht nur die Gestaltung von Schulgebäuden (Architektur, Ausstattung), sondern neben gesundheitsfördernden Essensangeboten auch den verbesserten Zugang zu Bewegungsräumen und eine Verbesserung der Interaktionsstrukturen zwischen Eltern, Schülerlnnen und Lehrkräften.

\section{Internet-Ressourcen}

Auf unserer Lehrbuch-Homepage (www.public-health-kompakt.de) finden Sie Hinweise auf weiterführende Literatur sowie Links zu themenrelevanten Studien und Institutionen. 